\title{
ENHANCEMENT OF ENTRAPMENT EFFICIENCY OF POROUS CHITOSAN SCAFFOLD FORMULATION BY THE ADDITION OF PEG 4000
}

\author{
Tarun Garg*, Arsh Chanana, Govind Sharma \\ Seth G.L.Bihani S.D.college of technical education, Sri Ganganagar
}

\section{INTRODUCTION}

Porous scaffold formulation is used for delivering the drug continuously to the target place but the major limitation face that poor entrapment efficiency. The objective of this study was enhancement of entrapment efficiency of porous chitosan scaffold formulation with the help of PEG 4000.

\section{MATERIALS AND METHODS}

\section{POROUS CHITOSAN SCAFFOLD PREPARATION}

Briefly different concentration of chitosan and PEG 4000 were dissolved in $0.2 \mathrm{M}$ glacial acetic acid and mixture of solution was poured into stainless steel mould and mould was stored in deep freezer at $-70^{\circ} \mathrm{C}$ for 5 days. After 5 days, mould was placed in lyophiliser (Martin Christ alpha 1-2 LD plus, Germany) for 3 days. After 3 days, blank porous chitosan scaffold was prepared. Gliclazide (GLZ) drug $(0.1 \%)$ was dissolved in ethanol and poured into porous matrix scaffold with the help of pipette using a post seeding method and drug loaded porous chitosan scaffold formulation was prepared [1].

\section{ENTRAPMENT EFFICIENCY OF SCAFFOLD FORMULATIONS}

Briefly, one piece of scaffold formulations was placed in $10 \mathrm{ml}$ centrifuge tube, which was filled with PBS (pH 7.4) solution, for 20 minute at $5000 \mathrm{rpm}$ centrifugation speed, in centrifuge machine (Remi R-8C). Absorbance of supernatant was checked at the $\lambda$ max. Value of $226.5 \mathrm{~nm}$ using UV-VIS spectrophotometer (Shimadzu UV 1700). PBS was used as a control. The \% entrapment efficiency of the scaffold formulations were calculated from the following equation. Entrapment efficiency $(\%)=$ $\mathrm{D}_{\mathrm{L}}-\mathrm{D}_{\mathrm{F}} / \mathrm{D}_{\mathrm{L}} \mathrm{x} 100$, where $\mathrm{D}_{\mathrm{L}}$ and $\mathrm{D}_{\mathrm{F}}$ are initial drug loaded $(\mathrm{mg})$ and free drug $(\mathrm{mg})$ respectively [2].

\section{RESULTS AND DISCUSSION}

\section{ENTRAPMENT EFFICIENCY OF GLICLAZIDE LOADED CHITOSAN SCAFFOLD FORMULATIONS}

6 different scaffold formulations were examined for entrapment efficiency. The effects of processing parameters such as polymer concentration, PEG 4000 concentration on the GLZ entrapment efficiency (EE) of scaffold formulations are presented in Table 1. It was observed that the EE of GLZ increased with an increase in the PEG 4000 concentration from 0 $2.5 \%$.

Table 1. Effects of various processing parameter on entrapment efficiency of GLZ in chitosan-PEG 4000 porous scaffold. Each data point represents a mean \pm standard deviation $(n=3)$.

\begin{tabular}{cccc}
\hline Formulation No. & Chitosan (\%) & PEG 4000 (\%) & Entrapment efficiency (\%) \\
\hline F1A & 1 & ----- & $60.52 \pm 2.49$ \\
F2A & 1 & 0.5 & $66.64 \pm 2.65$ \\
F3A & 1 & 1.0 & $69.46 \pm 2.03$ \\
F4A & 1 & 1.5 & $72.68 \pm 2.23$ \\
F5A & 1 & 2.0 & $74.76 \pm 1.98$ \\
F6A & 1 & 2.5 & $75.10 \pm 2.02$ \\
\hline
\end{tabular}




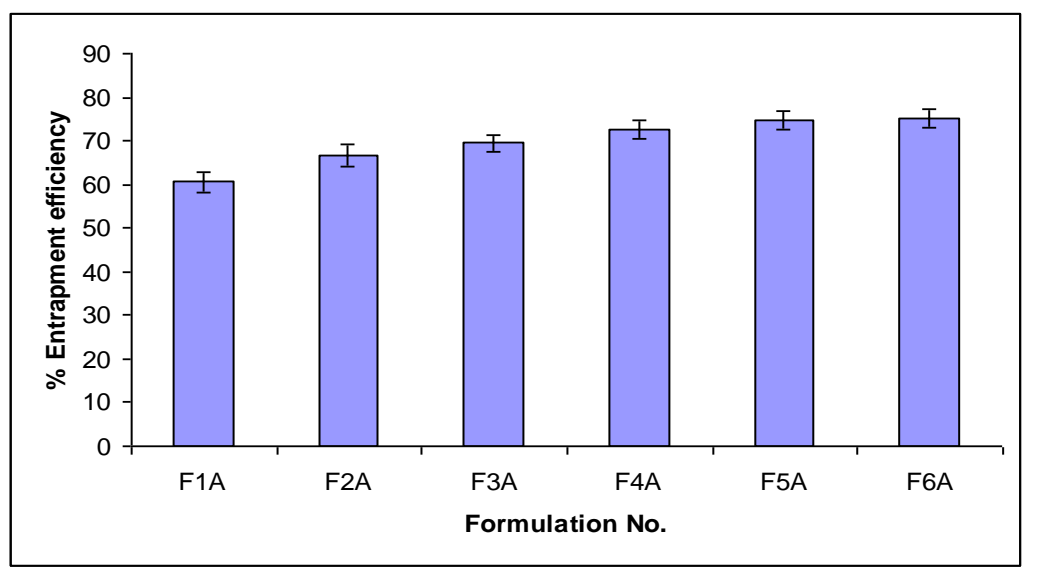

Fig 1. Entrapment efficiency of gliclazide loaded scaffold formulation (mean \pm S.D., $n=3$ )

Drug entrapment efficiency of scaffold formulations prepared with $1 \%$ chitosan with increasing concentration of PEG 4000 $(0-2.5 \%)$ showed variable drug entrapment efficiency. Increasing concentration of PEG 4000 from $0-2.5 \%$, increased $\%$ drug entrapment efficiency in scaffold formulations prepared with $1 \%$ chitosan. This is obviously due to the formation of high porous structure and more drugs were entrapped in it.

\section{CONCLUSIONS}

From this study, we concluded that, versatile carrier system i.e. porous scaffold formulation is effective for the delivery of gliclazide for long duration of time period because entrapment efficiency of porous chitosan scaffold increased by the addition of PEG 4000.

\section{REFERENCES}

[1] S.V. Madihally, H.W.T. Matthew, Porous chitosan scaffolds for tissue engineering, Biomaterials, 20, 1999, $1133-1142$.

[2] P.V. Devarajan, G.S. Sonavane, Preparation and in- vitro/in-vivo evaluation of gliclazide loaded eudragit nanoparticles as a sustained release carriers, Drug Development and Industrial Pharmacy,33,2007,101-111. 